\title{
Les plantes médicinales de la région montagneuse de Kahuzi-Biega en République démocratique du Congo : utilisation, accessibilité et consensus des tradipraticiens
}

\section{Chantal ShaLuKoma ${ }^{1,2}$ \\ Jan BOGAERT ${ }^{3}$ \\ Pierre Duez ${ }^{4}$ \\ Caroline STÉVIGNY2 \\ Célestin PongomBo ${ }^{5}$ \\ Marjolein VISSER ${ }^{1}$}

${ }^{1}$ Université libre de Bruxelles Service d'écologie du paysage et systèmes de production végétale CP 264/2, boulevard du Triomphe 1050 Bruxelles

Belgique

2 Université libre de Bruxelles Laboratoire de pharmacognosie, bromatologie et nutrition humaine CP 205/09, boulevard du Triomphe 1050 Bruxelles

Belgique

${ }^{3}$ Université de Liège, Gembloux Agro-Bio Tech

Unité biodiversité et paysage

2, passage des Déportés

5030 Gembloux

Belgique

\section{${ }^{4}$ Université de Mons}

Faculté de médecine et pharmacie Département de chimie

thérapeutique et pharmacognosie 20, place du Parc

7000 Mons

Belgique

${ }^{5}$ Université de Lubumbashi Faculté de médecine vétérinaire Département de pharmacologie, toxicologie et thérapeutique BP 1825, Lubumbashi République démocratique du Congo

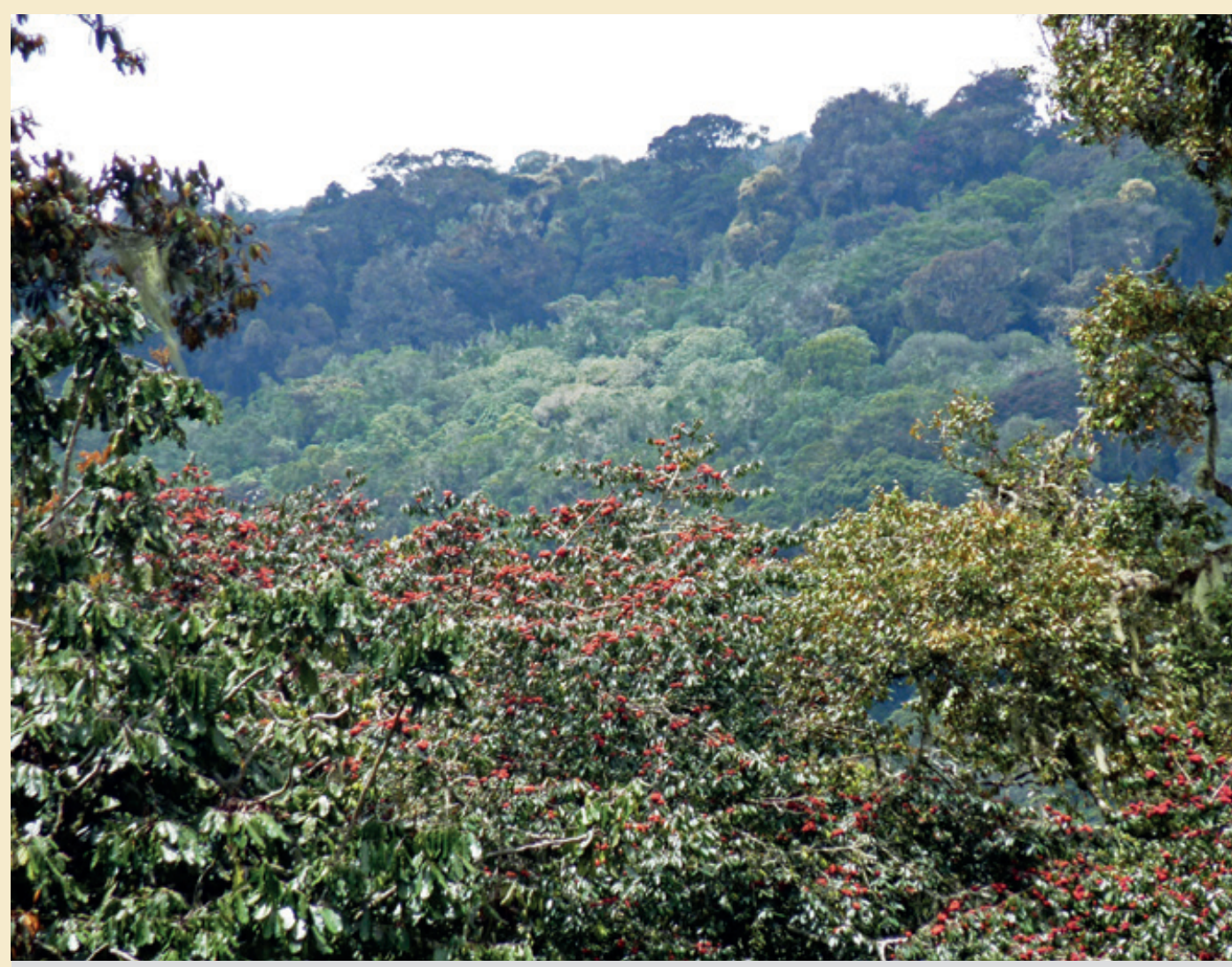

Photo 1.

Paysage de la forêt de montagne du Parc National de Kahuzi-Biega, Secteur Tshivanga. Photo C. Shalukoma. 


\section{RÉSUMÉ}

\section{LES PLANTES MÉDICINALES DE LA RÉGION MONTAGNEUSE DE KAHUZI- BIEGA EN RÉPUBLIQUE DÉMOCRATIQUE DU CONGO : UTILISATION, ACCESSIBILITÉ ET CONSENSUS DES TRADIPRATICIENS}

En ethnobotanique, des aspects comme l'influence de l'accessibilité d'une plante sur sa fréquence d'usage, le consensus entre les tradipraticiens autour des relations maladie-plante ainsi que le degré de fidélité d'une plante à une catégorie de maladies sont essentiels pour évaluer une tradition médicinale ; mais ils sont d'une interprétation difficile. Nous avons exploré ces aspects dans cette étude basée sur des entretiens semi-structurés avec 88 tradipraticiens issus des communautés Batwa, Havu, Shi et Tembo dans les localités situées à proximité de la forêt de montagne du Parc national de Kahuzi-Biega, en province du Sud-Kivu, à l'Est de la République démocratique du Congo. Il s'avère que 77 espèces végétales sont utilisées pour traiter les pathologies regroupées dans 18 catégories de maladies, dont les plus fréquentes sont les troubles digestifs et les infections. Les feuilles et les écorces sont les parties les plus utilisées dans les recettes majoritairement monoplantes et principalement préparées en solutions aqueuses. Bien que la corrélation entre l'accessibilité des plantes et leurs fréquences d'usage soit positive $(H=17,64 ; p<0,001)$, certaines plantes forestières pourtant moins accessibles connaissent des fréquences d'usage particulièrement élevées. Le facteur de consensus des tradipraticiens est globalement élevé, particulièrement pour les catégories de troubles musculo-squelettiques $(=0,83)$ et les infections $(=0,80)$. Toutes les catégories de maladies fidélisent au moins une plante malgré les taux globalement faibles. Bien qu'exploratoires, nos résultats suggèrent un certain ancrage d'une tradition médicinale au sein de communautés locales de la région. Cet ancrage suppose une bonne connaissance des plantes médicinales, une transmission intergénérationnelle des savoirs ainsi qu'une certaine collaboration entre les tradipraticiens. Plus d'études sont nécessaires pour évaluer davantage ces différents aspects.

Mots-clés : ethnobotanique quantitative, forêt, médecine traditionnelle, Parc de Kahuzi-Biega, tradition médicinale, République démocratique du Congo.

\section{ABSTRACT}

\section{MEDICINAL PLANTS OF THE MOUNTAIN REGION OF KAHUZI-BIEGA IN THE DEMOCRATIC REPUBLIC OF CONGO: USES, ACCESSIBILITY AND CONSENSUS AMONG TRADITIONAL THERAPISTS}

For ethnobotanists, aspects such as the influence of a plant's accessibility on its frequency of use, consensus among traditional therapists over the relationships between plants and diseases and the degree of fidelity to a plant for a given category of ailments are essential to assess a medicinal tradition, but difficult to interpret. We explored these aspects through a study based on semi-structured interviews with 88 traditional therapists from the Batwa, Havu, Shi and Tembo communities near the montane forests of the Kahuzi-Biega National Park, in South Kivu Province in eastern DRC. Our survey showed that 77 plant species are used to treat pathologies grouped into 18 categories of ailments, the most frequent being digestive disorders and infections. Leaves and bark are the parts most frequently used, usually in recipes involving a single plant and mainly prepared as aqueous solutions. Although there is a positive correlation between the accessibility of plants and the frequency of their use $(H=17.64 ; p<0.001)$, some less accessible forest plants have very high use frequencies. Consensus between traditional therapists is high overall, especially for musculo-skeletal disorders $(=0.83)$ and infections $(=0.80)$. Although the degree of fidelity to a plant for a given category of ailments is low overall, it is always high for at least one plant in all categories. Our results, although exploratory, suggest that certain medicinal traditions are well rooted in the region's local communities. This assumes a good local knowledge of medicinal plants, intergenerational transmission of that knowledge and a degree of cooperation between traditional therapists. Further studies are needed to assess these different aspects with greater precision.

KeYwords: Quantitative ethnobotany, forest, traditional medicine, Kahuzi-Biega Park, medicinal tradition, Democratic Republic of Congo.
RESUMEN

\author{
PLANTAS MEDICINALES DE LA REGIÓN \\ MONTAÑOSA DE KAHUZI-BIEGA EN LA \\ REPÚBLICA DEMOCRÁTICA DEL CONGO: \\ USO, ACCESIBILIDAD Y CONSENSO \\ ENTRE TERAPEUTAS TRADICIONALES
}

En etnobotánica, la influencia de la accesibilidad de una planta en su frecuencia de uso, el consenso entre terapeutas tradicionales sobre las relaciones enfermedad-planta y el grado de fidelidad hacia una planta para tratar una categoría de enfermedades son aspectos esenciales, aunque de difícil interpretación, para evaluar una tradición medicinal. En este estudio se indagan estos aspectos mediante entrevistas semiestructuradas a 88 terapeutas tradicionales de los pueblos Batwa, Havu, Shi y Tembo en poblados ubicados cerca del bosque de montaña del Parque Nacional de Kahuzi-Biega, provincia de Sud-Kivu, en el este de la República Democrática del Congo. Se observó que se utilizan 77 especies vegetales para tratar las enfermedades, agrupadas en 18 categorías, siendo las más frecuentes los trastornos digestivos y las infecciones. Hojas y cortezas son las partes más empleadas en recetas mayoritariamente a base de una única planta que se suele preparar en solución acuosa. Aunque la correlación entre accesibilidad de las plantas y frecuencia de uso es positiva $(H=17,64 ; p<0,001)$, ciertas plantas forestales menos accesibles muestran frecuencias de uso especialmente altas. El factor de consenso entre terapeutas tradicionales es globalmente alto, especialmente en las categorías de trastornos musculoesqueléticos $(=0,83)$ e infecciones $(=0,80)$. Todas las categorías de enfermedades fidelizan al menos una planta, a pesar de tasas globalmente bajas. Nuestros resultados, aunque de carácter exploratorio, sugieren un cierto arraigo de la tradición medicinal en las comunidades locales de la región. Dicho arraigo supone un buen conocimiento de las plantas medicinales, una transmisión intergeneracional de conocimientos y cierta colaboración entre terapeutas tradicionales. Es necesario ampliar los estudios para evaluar más detalladamente todos estos aspectos.

Palabras clave: Etnobotánica cuantitativa, bosque, medicina tradicional, Parque Kahuzi-Biega, tradición medicinal, República Democrática del Congo. 


\section{Introduction}

Les forêts d'Afrique offrent des moyens de subsistance à près de 60 millions de personnes qui y vivent ou résident à proximité (nourriture, pharmacopée, combustibles, fibres, produits forestiers non ligneux) (De Wasseige et al., 2014). Pour les besoins de soins de santé primaires, l'utilisation des plantes médicinales tient encore une place de choix sur le continent. Cependant, l'accès à ces plantes utiles devient un problème croissant à cause de la dégradation progressive de la végétation naturelle et l'érosion des connaissances traditionnelles (Vandebroek et Balick, 2012 ; Malan et al., 2015). L'ethnobotanique est une discipline récente, décrite par Turner (1988) comme une science des interactions entre les humains et les plantes. Elle documente et analyse la connaissance des plantes dans le contexte culturel local (Heinrich et al., 1998). Comme le soulignent Pirker et al. (2012), la connaissance des plantes médicinales s'acquiert à travers des pratiques et des croyances qui évoluent de génération en génération, et ce grâce à une transmission culturelle basée sur les relations entre les humains et leur environnement. Cet empirisme est à la base de l'homogénéité des connaissances locales pour le traitement des catégories de maladies répandues dans une zone donnée (Heinrich et al., 1998). Cette homogénéité suggère une tradition médicinale établie ayant résisté aux différentes perturbations internes et externes aux communautés. Comment alors évaluer cette homogénéité de connaissances?

Dans le contexte de dégradation des ressources naturelles, particulièrement en République démocratique du Congo (RDC), on peut soupçonner une érosion des connaissances des tradipraticiens et, par conséquent, la perte de l'homogénéité de ces connaissances.

Cette étude a voulu montrer que la convergence de connaissances locales peut aller au-delà de l'appartenance ethnogéographique de tradipraticiens et que ces connaissances homogènes peuvent se maintenir malgré les différents contextes socio-écologiques dans lesquels ils vivent. La méthode pertinente pour aborder cette question a été celle de la quantification des usages locaux des plantes médicinales (Heinrich et al., 1998). L'ethnobotanique quantitative permet de transformer le savoir traditionnel local en valeur quantifiable d'utilisation relative (Wong et al., 2001) et de dégager des tendances par l'analyse statistique (Phillips, 1996 ; Nzuki et al., 2013). Les approches basées sur la notion de consensus d'informateurs sont indiquées pour une évaluation ethnobotanique intra-culturelle. Les plus utilisées sont le facteur de consensus des informateurs (Trotter et Logan, 1986) et la fidélité des plantes aux catégories de maladies (Friedman et al., 1986).

En RDC, la médecine traditionnelle vient d'être réorganisée par l'arrêté n 1250/62/2002. Celui-ci reconnaît tout phytothérapeute enregistré et reconnu dans sa communauté (CNOM, 2015). Parmi les régions forestières du pays, celle de Kahuzi-Biega est connue pour sa richesse naturelle et culturelle (ICCN, 2009) bien que très peu d'études existent sur son potentiel ethno-médicinal en rapport avec les usages des plantes médicinales. Le présent travail contribuera à enrichir les connaissances existantes, surtout en quantifiant les usages médicinaux des plantes de la zone. Les travaux précédents réalisés dans la région avaient permis de constituer des catalogues de plantes médicinales, souvent rudérales ou cultivées (Defour, 1995 ; Schneider, 1996 ; Balagizi et al., 2007). Mangambu et al. (2012) ont étudié l'ethnomédecine et la conservation des ptéridophytes autour de la forêt de montagne du Parc national de Kahuzi-Biega (PNKB). Autour de cette forêt de montagne du PNKB, la typologie des tradipraticiens est basée sur leur spécialisation et non pas sur leur appartenance ethnique ou géographique (données non publiées). Ce dernier résultat suggère que l'ensemble des ethnies pourrait être considéré comme un seul groupe culturel. Il est donc opportun d'en évaluer le degré d'homogénéité intra-culturelle de connaissances.

\section{Matériel et méthode}

\section{Récolte des données}

Les données ethnobotaniques ont été récoltées entre 2009 et 2010 auprès de 88 tradipraticiens localisés dans 33 villages situés aux limites de la forêt de montagne du PNKB (figure 1). Cette étude n'a pris en compte que les tradipraticiens reconnus au sein des communautés Batwa, Havu, Shi et Tembo vivant dans les localités voisines de la forêt de montagne du Parc national de Kahuzi-Biega.

La récolte des données a été précédée par une préenquête afin de connaître la zone et d'identifier les tradipraticiens spécialistes. Les données issues de l'enquête proprement dite ont été soumises à la vérification et à la validation auprès des tradipraticiens rencontrés dans différentes localités. Cela nous avait permis de nous rassurer sur la qualité des données récoltées et surtout de limiter les erreurs liées à la désignation des plantes et des maladies par leurs noms vernaculaires. Les entretiens étaient semi-structurés et agencés comme suit : identité des tradipraticiens (nom, sexe, ethnie, localisation géographique) ; principales maladies pour lesquelles ils sont consultés ; pratiques basées sur les plantes utilisées, en mentionnant les organes préparés, les types de recettes médicinales, les modes de préparation et les voies d'administration. Les recettes ont été répertoriées selon les plantes et les organes utilisés. La préenquête menée avait permis d'aboutir à un échantillonnage exhaustif des tradipraticiens, peu nombreux dans les territoires administratifs de Kabare et Kalehe. Les entretiens étaient individuels et menés en langues shi et swahili. Chaque tradipraticien a été visité au moins deux fois et a participé à au moins une sortie d'identification des espèces médicinales en milieux naturels dans la zone. Aucun patient n'a été interviewé lors des enquêtes. Les échantillons de plantes étaient collectés en deux exemplaires. Des spécimens entiers étaient généralement recueillis pour les petites plantes herbacées. Les caractéristiques botaniques et ethnobotaniques ainsi que les noms vernaculaires des plantes étaient notés sur les échantillons déposés aux herbariums de Lwiro (RDC) et du Jardin botanique de Meise (Belgique). 


\section{Analyse des données}

\section{Plantes médicinales et pratiques médicales associées}

Les pratiques médicales étudiées ont concerné les plantes utilisées, les parties prélevées, les modes de préparation et les voies d'administration des drogues. Les maladies citées par les tradipraticiens ont été regroupées en catégories selon la classification standard des usages des plantes médicinales (Cook, 1995 ; Gruca et al., 2014). Les auteurs précités présument que les catégories correspondant à un grand nombre des mentions reflètent les maux qui affectent le plus la population.

\section{Accessibilité et fréquences d'usage des plantes médicinales}

L'accessibilité des plantes a été liée aux types d'habitats. Elle a été traduite sur une échelle de valeurs allant de 1 à 4 comme suit : plantes rudérales, de jachère, des bords de route (4) ; plantes de jardin (3) ; forêt secondaire (2) ; forêt primaire (1). Les habitats ont été principalement définis sur la base des lieux de récolte des espèces lors de nos missions sur le terrain. Ensuite, ces données ont été confrontées à la littérature via la base de données de Plant Resources of Tropical Africa (Prota4U, 2015) et aux avis d'experts botanistes et écologues. Le test non paramétrique de Spearman a permis de mesurer la corrélation entre les fréquences d'usage des plantes et les nombres de mentions des valeurs d'accessibilité pour chaque plante. La fréquence d'usage correspond au nombre de fois qu'une plante a été citée pour une catégorie donnée de maladies, dans une matrice de variables quantitatives de 77 plantes $x$ 18 classes de maladies.

\section{Consensus d'informateurs et fidélité de plantes médicinales}

Les techniques les plus populaires (indices) pour mesurer l'importance culturelle des plantes sont basées sur le consensus d'informateurs, qui reflète le degré d'accord entre les différentes personnes interrogées concernant l'utilisation d'une ressource donnée (Albuquerque, 2009). Dans cette étude, le consensus a été dégagé par deux approches, à savoir le facteur de consensus d'informateurs $\left(F_{\mathrm{ci}}\right)$ et l'indice de fidélité $(I F)$ des plantes. Le $F_{\text {ci }}$ pris en compte dans cette étude est l'indice qui permet d'évaluer la consistance de la connaissance des tradipraticiens sur la base des maladies qu'ils traitent et des plantes qu'ils utilisent (Trotter et Logan, 1986 ; Molares et Ladi, 2009 ; Musa et al., 2011). Un $F_{\text {ci }}$ élevé pour une catégorie donnée de maladies suggère un consensus entre les tradipraticiens par rapport aux plantes

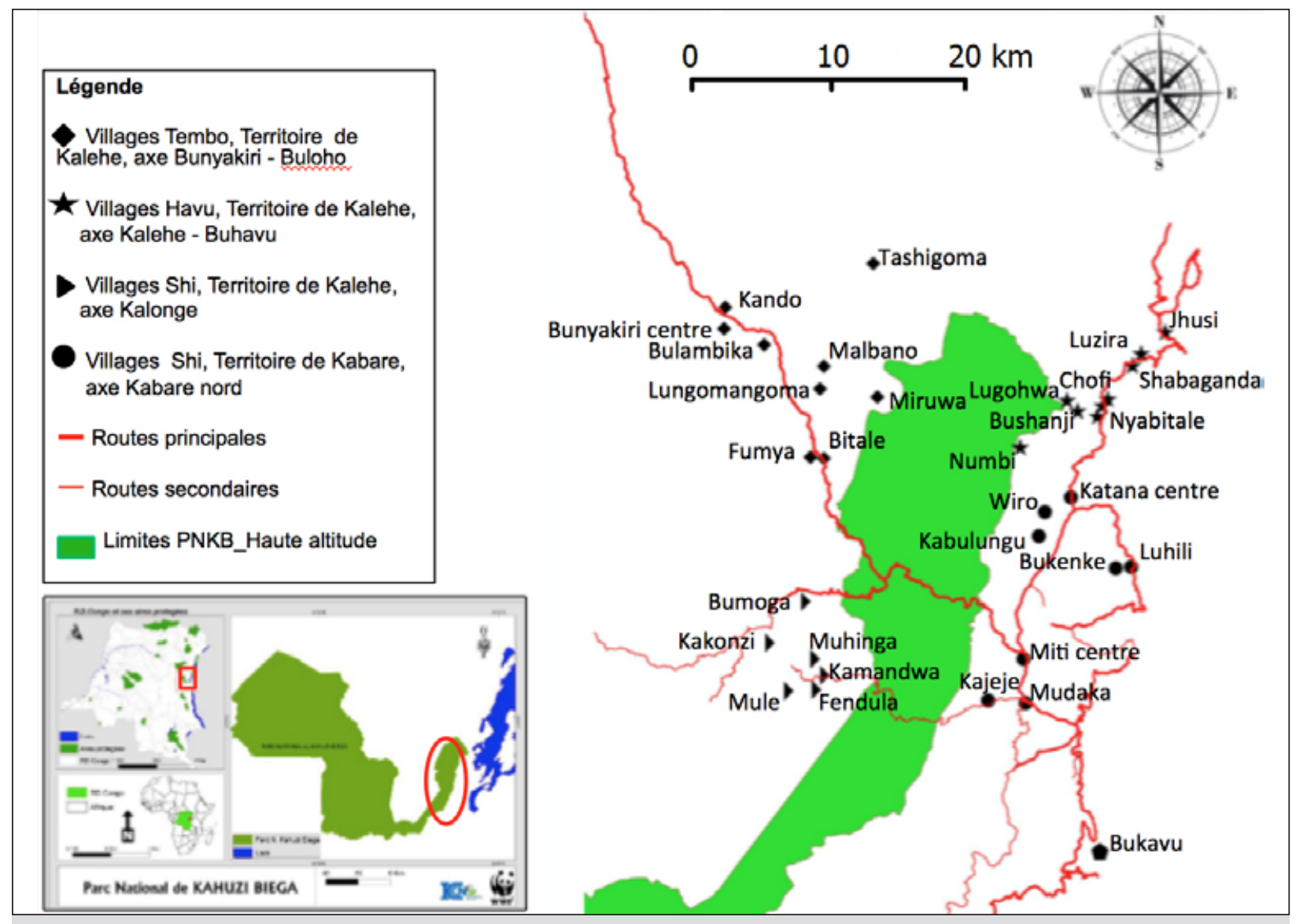

Figure 1.

Parc national de Kahuzi-Biega : site d'étude (encadré en noir) (Shalukoma, 2015). 
qu'ils utilisent pour les maladies qu'ils traitent ; mais également que cette catégorie renferme des maladies fréquentes dans le milieu. Il rend compte, s'il est élevé, d'une tradition bien définie liée aux plantes médicinales associées à ces catégories de maladies (Heinrich et al., 1998 ; Pirker et al., 2012). Ce facteur de consensus d'informateurs exprime leur «taux d'approbation » sur la base du nombre des mentions d'une catégorie de maladies et le nombre total de plantes utilisées pour soigner des maladies regroupées dans la catégorie donnée. L'indice est calculé par catégorie de maladies selon la formule suivante (Trotter et Logan, 1986 ; Molares et Ladi, 2009 ; Musa et al., 2011) :

$F_{c i}=\frac{n_{r}-n_{t}}{n_{r}-1}$

où $n_{\mathrm{r}}$ désigne le nombre des mentions des maladies de la

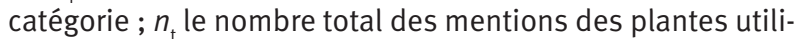
sées pour soigner les maladies identifiées dans la catégorie.

Une mention signifie une citation, par un informateur donné, d'une maladie (appartenant à une catégorie donnée) associée à une plante ou une combinaison de plantes pour la traiter. Si un informateur associe la même maladie à différentes espèces ou combinaisons d'espèces, le nombre de mentions attribué à cette maladie par cet informateur égale le nombre de traitements différents que cet informateur applique.

Le nombre de mentions pour une catégorie de maladies est la somme totale de toutes les citations attribuées à toutes les maladies de cette catégorie par les différents informateurs.

Le nombre d'espèces associé à une maladie (appartenant à une catégorie donnée) est le total des espèces mentionnées au moins une fois pour traiter cette maladie, que cela soit en association avec d'autres espèces ou non, par le même ensemble d'informateurs.

En théorie, le $F_{\text {ci }}$ peut prendre des valeurs négatives mais, dans la pratique, la majorité des traitements d'une maladie donnée par les plantes se passe avec une recette monoplante, de sorte que le nombre de mentions augmente généralement aussi ou plus vite que le nombre d'espèces.

Le $F_{c i}$ varie donc généralement entre 0 et 1 . Une valeur faible (proche de 0 ) indique que les informateurs ne sont pas en accord sur les plantes utilisées pour soigner les maladies d'une catégorie donnée. Dans cette étude, le seuil empirique de $F_{c i} \geq 0,70$ a été choisi pour suggérer que le consensus entre les tradipraticiens est élevé. Ce seuil représente la valeur médiane de l'ensemble des $F_{c i}$ de catégories de maladies identifiées. En dessous de ce seuil, le consensus entre tradipraticiens est considéré comme moyen $\left(F_{\mathrm{ci}}\right.$ entre 0,70 et 0,50$)$ ou faible $\left(F_{c i}<0,50\right)$.

L'indice de fidélité $(I F)$ aide à apprécier l'intensité de la relation que les tradipraticiens établissent entre une plante médicinale et son rôle dans une catégorie donnée de maladies. Cet indice est basé sur le pourcentage des tradipraticiens qui ont confirmé l'usage d'une plante dans le traitement d'une catégorie de maladies. Il est calculé par la formule suivante (Friedman et al., 1986 ; Giday et al., 2009 ; Ugulu, 2012) :
$I F(\%)=\frac{I p}{I u} \times 100$

où Ip est le nombre d'informateurs ayant utilisé l'espèce donnée pour traiter une catégorie donnée de maladies ; lu est le nombre total des tradipraticiens ayant cité cette même espèce au moins une fois pour toutes les catégories de maladies. On obtient donc une valeur pour chaque paire constituée de catégorie de maladies et d'espèces.

\section{Statistiques inductives}

Le test de corrélation de Spearman (Sokal et Rohlf, 2012) a permis d'évaluer la dépendance entre le consensus des tradipraticiens (une valeur par catégorie de maladies) et le nombre de mentions de chaque catégorie de maladies. Le test de Kruskal-Wallis (Sokal et Rohlf, 2012) a été utilisé pour tester si les médianes des fréquences d'usage des populations observées d'une plante donnée diffèrent significativement en fonction de leur accessibilité. Les tests non paramétriques ont été réalisés dans Excel.

\section{Résultats}

\section{Plantes médicinales et pratiques médicales associées}

Un total de 77 plantes médicinales a été identifié auprès des tradipraticiens rencontrés (tableau I). Elles se distribuent en 42 familles et 73 genres. Les familles les plus représentées en nombre d'espèces sont les Asteraceae (14\%), Euphorbiaceae (9\%), Fabaceae et Rubiaceae (6 \%). Parmi les espèces inventoriées, les espèces de forêt secondaire représentent $56 \%$ tandis que les espèces de la forêt primaire en recouvrent $23 \%$. Les espèces rudérales et les espèces plantées représentent respectivement $17 \%$ et $4 \%$ du total. Les espèces forestières dominent la pharmacopée des spécialistes autour du PNKB.

Les parties des plantes les plus utilisées sont les feuilles (47\%), les écorces (21\%) et les racines (12\%). Les autres parties, faiblement utilisées (20\%), sont les fruits, la sève, les noix, les fleurs, les plantes entières, les tiges et les graines. Les écorces sont massivement prélevées. Les troncs d'arbre écorcés sont très présents dans différents habitats de la forêt (photos $2 a$ et $2 b$ ). Les tradipraticiens en constituent des stocks après séchage (photo 3 ). La préparation de solutions (décoction, expression, infusion, macération) constitue le mode le plus fréquent ( $80 \%$ ), le principal solvant étant l'eau, parfois le jus de banane ou le miel. Sont peu fréquentes, les préparations sous forme de cendres (7\%), de poudres (6\%) et de pommades (1\%). Les plantes utilisées sans préparation sont également moins communes (6\%). Les principaux modes d'administration de médicaments sont la voie orale (45\%), l'application cutanée (15\%) et la scarification (10\%). L'inhalation et les bains sont peu fréquents (30\%) dans la zone. Un total de 299 recettes médicinales ont été inventoriées, dont la majorité sont réalisées à base d'une seule plante (75\%). Les recettes préparées à base de plusieurs plantes sont beaucoup plus caractérisées par les plantes forestières associées à celles des jachères. 
Tableau I.

Liens entre plantes médicinales (classées par ordre alphabétique au sein des classes d’accessibilité croissantes), pratiques médicales et catégories de maladies.

\begin{tabular}{|c|c|c|c|c|c|c|c|c|}
\hline Familles & Espèces & $\begin{array}{l}\text { Valeurs } \\
\text { d'acces- } \\
\text { sibilité }\end{array}$ & $\begin{array}{l}\text { Fréquences } \\
\text { absolues } \\
\text { d'usage }\end{array}$ & $\begin{array}{l}\text { Fréquences } \\
\text { relatives } \\
\text { d'usage (\%) }\end{array}$ & $\begin{array}{l}\text { Parties } \\
\text { utilisées }\end{array}$ & $\begin{array}{l}\text { Modes de } \\
\text { préparation }\end{array}$ & $\begin{array}{l}\text { Voies } \\
\text { d'admini- } \\
\text { stration }\end{array}$ & $\begin{array}{l}\text { Catégories } \\
\text { de maladies }\end{array}$ \\
\hline Euphorbiaceae & Alchornea cordifolia (Schumach.) & 1 & 2 & 0,5 & $\mathrm{f}, \mathrm{fl}$ & dec & vo, scar, mas, bc & cult \\
\hline Sapotaceae & Autranella congolensis A. Chev. \& De Wild. & 1 & 2 & 0,5 & $e, r$ & dec, cen, pou & vo, ac & imf, poi \\
\hline Meliaceae & Carapa grandiflora Sprague & 1 & 11 & 2,7 & f, e, gr & dec, pou & vo, mas, bs & inf, gen, ner, gro, dig cult \\
\hline Rubiaceae & Coffea kivuensis Lebrun & 1 & 3 & 0,7 & f, gr & inf, pou & vo & inf, gen, cir \\
\hline Meliaceae & Entandrophragma excelsum Sprague & 1 & 6 & 1,5 & $\mathrm{f}, \mathrm{r}, \mathrm{e}$ & dec & vo & inf, san, gen, met, dig \\
\hline Clusiaceae & Lebrunia bushaie Staner & 1 & 2 & 0,5 & $f$ & dec & $\mathrm{ac}$ & cut \\
\hline Fabaceae & Millettia psilopetala Harms & 1 & 4 & 1,0 & f, e & dec, cen & vo, bc, scar, mas & inf, cut, cir, cult \\
\hline Fabaceae & $\begin{array}{l}\text { Newtonia buchananii (Baker) } \\
\text { G.C.C. Gilbert \& Boutique }\end{array}$ & 1 & 2 & 0,5 & $f$ & dec & vo & ner \\
\hline Chrysobalanaceae & Parinari excelsa Sabine & 1 & 5 & 1,2 & $f, e, r, f r$ & dec, pou & vo & inf, met, ner, dig \\
\hline Fabaceae & Piptadeniastrum africanum (Hook. f.) Brenan & 1 & 1 & 0,2 & $e, f$ & dec & vo & san \\
\hline Apocynaceae & Pleiocarpa pycnantha Stapf & 1 & 3 & 0,7 & $e, f$ & dec, pou & vo & poi, dig \\
\hline Rosaceae & Prunus africana (Hook. f.) Kalkman & 1 & 3 & 0,7 & f, e & dec & vo & gen, dig \\
\hline Myrsinaceae & Rapanea melanophloeos Mez & 1 & 1 & 0,2 & $f$ & dec & vo & inf \\
\hline Olacaceae & Strombosia scheffleri Engl. & 1 & 2 & 0,5 & f, e & pou, inf & scar, mas, vo & ner \\
\hline Clusiaceae & Symphonia globulifera L. f. & 1 & 1 & 0,2 & $f, e, f l$ & dec & vo & san \\
\hline Myrtaceae & Syzygium cordatum Hochst. & 1 & 9 & 2,2 & f, e & dec & vo & gen, poi, dig \\
\hline Myrtaceae & Syzygium guineense DC. & 1 & 1 & 0,2 & $f$ & dec & vo & dig \\
\hline Tiliaceae & Triumfetta cordifolia A. Rich. & 1 & 1 & 0,2 & $f$ & dec & scar, mas, bc & cult \\
\hline Alangiaceae & Alangium chinense (Lour.) Harms & 2 & 1 & 0,2 & e & dec & vo & ner \\
\hline Fabaceae & Albizia gummifera C.A. Sm & 2 & 3 & 0,7 & $f$ & exp, dec, pou & inst, bc, scar, mas & sen \\
\hline Euphorbiaceae & Alchornea hirtella Benth. & 2 & 2 & 0,5 & $f$ & dec, cen & vo, scar & cir, ner \\
\hline Apocynaceae & Baissea multiflora A.DC. & 2 & 7 & 1,7 & $f, e, r$ & dec, exp, inf & vo, ac & inf, imf, ble, res, en, syng \\
\hline Basellaceae & Basella alba L. & 2 & 5 & 1,2 & $f$ & dec, inf & vo & imf, gro \\
\hline Begoniaceae & Begonia meyeri-Johannis Engl. & 2 & 9 & 2,2 & f, pe & dec, exp & vo, scar, mas, ac & gen, gro, mus, cult, dig \\
\hline Euphorbiaceae & Bridelia micrantha Baill. & 2 & 6 & 1,5 & f, e, gr & dec, cen, pou & vo, bc, asp, scar & gen, cir, dig, cult \\
\hline Burseraceae & Canarium schweinfurtii Engl. & 2 & 5 & 1,2 & f, e, r, s & dec, cen, mac & vo, inh & san, poi, ner \\
\hline Lamiaceae & Clerodendrum welwitschii Gürke & 2 & 4 & 1,0 & $f, r$ & dec & vo & imf, gen, cult \\
\hline Cyatheaceae & Cyathea manniana Hook. & 2 & 2 & 0,5 & $f, r$ & dec & vo & gen, gro \\
\hline Myrsinaceae & Embelia schimperi Vatke & 2 & 6 & 1,5 & f & dec, inf, sp & vo & gen, ner, gro, dig \\
\hline Musaceae & Ensete ventricosum (Welw.) Cheesman & 2 & 8 & 2,0 & $f, r$ & dec & vo & inf, san, gen, ner, gro, dig \\
\hline Fabaceae & Erythrophleum guineense G. Don & 2 & 2 & 0,5 & $f$ & dec & vo & san, gro \\
\hline Moraceae & Ficus oreodryadum Mildbr. & 2 & 4 & 1,0 & f, e & dec, exp & vo, ac & cut, ner \\
\hline Moraceae & Ficus thonningii Blume & 2 & 1 & 0,2 & $f$ & $\mathrm{sp}$ & vo & inf \\
\hline Rubiaceae & Galiniera saxifraga (A. Rich.) Bridson & 2 & 1 & 0,2 & f & dec & scar, mas, bc & cult \\
\hline Rhamnaceae & Gouania longispicata Engl. & 2 & 7 & 1,7 & f, s, gr, t & dec, sp & vo & nut, gen, met, gro \\
\hline Rubiaceae & Hallea rubrostipulata (K. Schum.) J.-F. Leroy & 2 & 3 & 0,7 & f, e & dec & vo & gen, gro, dig \\
\hline Clusiaceae & Harungana montana Spirl. & 2 & 9 & 2,2 & $f, e, r$ & dec & vo & inf, cut, san, gen, met, dig \\
\hline Convolvulaceae & Ipomoea involucrata P. Beauv. & 2 & 3 & 0,7 & f & $\exp , \exp$ & $\mathrm{ac}$ & cut \\
\hline Oleaceae & Jasminum abyssinicum $\mathrm{R}$. Br. & 2 & 1 & 0,2 & $f$ & inf & vo & dig \\
\hline Asteraceae & Lactuca attenuata Stebbins & 2 & 5 & 1,2 & $\mathrm{f}, \mathrm{fl}$ & dec, exp & vo, mas, asp, bc & gro, mus, cult \\
\hline Campanulaceae & Lobelia giberroa Hemsl. & 2 & 2 & 0,5 & $\mathrm{f}, \mathrm{fl}$ & dec & vo & res \\
\hline Euphorbiaceae & Macaranga kilimandscharica Pax & 2 & 2 & 0,5 & f & dec & vo, bc, scar, mas & ner, cult \\
\hline Myrsinaceae & Maesa lanceolata Forssk. & 2 & 15 & 3,7 & f, e & $\begin{array}{l}\text { dec, pou, exp, } \\
\text { pom, sp }\end{array}$ & vo, ac, mas & poi, ner, mus, cult \\
\hline Asteraceae & Mikania cordata (Burm.f.) B.L. Rob. & 2 & 12 & 2,9 & $\mathrm{f}, \mathrm{fl}$ & dec, exp & vo, mas & $\begin{array}{l}\text { imf, san, gen, ner, } \\
\text { gro, mus, dig }\end{array}$ \\
\hline
\end{tabular}




\begin{tabular}{|c|c|c|c|c|c|c|c|c|}
\hline Familles & Espèces & $\begin{array}{l}\text { Valeurs } \\
\text { d'acces- } \\
\text { sibilité }\end{array}$ & $\begin{array}{l}\text { Fréquences } \\
\text { absolues } \\
\text { d'usage }\end{array}$ & $\begin{array}{l}\text { Fréquences } \\
\text { relatives } \\
\text { d'usage (\%) }\end{array}$ & $\begin{array}{l}\text { Parties } \\
\text { utilisées }\end{array}$ & $\begin{array}{l}\text { Modes de } \\
\text { préparation }\end{array}$ & $\begin{array}{l}\text { Voies } \\
\text { d'admini- } \\
\text { stration }\end{array}$ & $\begin{array}{l}\text { Catégories } \\
\text { de maladies }\end{array}$ \\
\hline Moraceae & Milicia excelsa (Welw.) C.C. Berg & 2 & 2 & 0,5 & e & dec & vo & imf, sym \\
\hline Urticaceae & Myrianthus holstii Engl. & 2 & 5 & 1,2 & $f, r$ & dec & vo, scar, mas & cut, cir, dig, cult \\
\hline Euphorbiaceae & Neoboutonia africana (Hook. f.) Kalkman & 2 & 4 & 1,0 & f, e & dec & vo & ner, dig \\
\hline Euphorbiaceae & Neoboutonia macrocalyx Pax & 2 & 3 & 0,7 & $\mathrm{f}, \mathrm{e}, \mathrm{fr}$ & dec, inf & vo & Poi, inf \\
\hline Asclepiadaceae & Periploca linearifolia Quart.-Dill. \& A. Rich. & 2 & 2 & 0,5 & $f$ & dec, sp & vo & gen, gro \\
\hline Piperaceae & Piper capense L. f. & 2 & 10 & 2,4 & $\mathrm{f}, \mathrm{fr}, \mathrm{r}$ & inf & $\begin{array}{l}\text { vo, scar, mas, } \\
\text { bc, asp }\end{array}$ & san, res, gen, dig, cult \\
\hline Araliaceae & Polyscias fulva Hutch. \& Dalziel & 2 & 7 & 1,7 & $\mathrm{f}, \mathrm{fl}, \mathrm{e}, \mathrm{r}$ & dec, inf, cen & vo, mas, ac, bv & ner, mus, cult \\
\hline Rubiaceae & Rubia cordifolia Hochst. ex A. Rich. & 2 & 2 & 0,5 & f & dec, exp & vo, ac & cut, ble \\
\hline Apocynaceae & Tabernaemontana johnstonii (Stapf) Pichon & 2 & 14 & 3,4 & f, e, r & dec, sp, exp & vo, ac, bb & inf, cut, gen, gro, dig, cult \\
\hline Ulmaceae & Trema Orientalis (L.) Blume & 2 & 12 & 2,9 & f, e & dec, cen, & $\begin{array}{l}\text { vo, ac, mas } \\
\text { exp, pom }\end{array}$ & $\begin{array}{l}\text { inf, imf, ble, san, res, } \\
\text { mus, dig, cult }\end{array}$ \\
\hline Rubiaceae & Tricalysia niamniamensis Schweinf. ex Hiern & 2 & 2 & 0,5 & f, e & dec, mac & $\mathrm{ac}$ & cut \\
\hline Urticaceae & Urera hypselodendron Engl. & 2 & 5 & 1,2 & $\mathrm{f}, \mathrm{t}, \mathrm{fl}$ & dec, exp & ac, vo & cut, imf, ble, cir, gro \\
\hline Asteraceae & Vernonia amygdalina Delile & 2 & 2 & 0,5 & f, e & dec & vo & met \\
\hline Asteraceae & Vernonia hochstetteri Sch. Bip. ex Hochst. & 2 & 6 & 1,5 & f, e, r & dec, inf & vo, bs & inf, gen, met, ner, dig \\
\hline Asteraceae & Vernonia kirungae R.E. Fr. & 2 & 4 & 1,0 & $f$ & dec, exp & vo, bc & inf, dig, cult \\
\hline Rutaceae & $\begin{array}{l}\text { Zanthoxylum lemairei (De Wild.) } \\
\text { P. G. Waterman }\end{array}$ & 2 & 3 & 0,7 & e & dec, cen & vo, ac & inf \\
\hline Rutaceae & $\begin{array}{l}\text { Zanthoxylum gilletii Oliv. (De Wild.) } \\
\text { P. G. Waterman }\end{array}$ & 2 & 10 & 2,4 & $e, r, f$ & dec, cen & $\begin{array}{l}\text { vo, ac, bc, s } \\
\text { scar, ma }\end{array}$ & $\begin{array}{l}\text { inf, gen, syn, poi, } \\
\text { gro, dig, cult }\end{array}$ \\
\hline Aloaceae & Aloe barbadensis Mill. & 3 & 12 & 2,9 & f, s & dec, sp, exp & vo, ac & $\begin{array}{l}\text { inf, cut, san, res, gen, } \\
\text { cir, poi, dig, imf }\end{array}$ \\
\hline Moringaceae & Moringa oleifera Lam. & 3 & 2 & 0,5 & r, gr & dec & vo & met, cir \\
\hline Lauraceae & Persea americana Mill. & 3 & 7 & 1,7 & f, no & dec & vo & cir, dig \\
\hline Asteraceae & Ageratum conyzoides $\mathrm{L}$. & 4 & 7 & 1,7 & $f$ & dec, exp & vo, ac & imf, syn, gro, dig, cult \\
\hline Asteraceae & Anisopappus africanus Oliv. \& Hiern & 4 & 6 & 1,5 & f & dec, exp & vo, inst & dig, ner, res \\
\hline Asteraceae & Bidens pilosa $\mathrm{L}$. & 4 & 14 & 3,4 & $\mathrm{f}$, fl, pe & dec, exp & vo & $\begin{array}{l}\text { imf, san, sen, sym, ner, } \\
\text { poi, gro, dig, cult }\end{array}$ \\
\hline Asteraceae & Conyza aegyptiaca (L.) Aiton & 4 & 8 & 2,0 & f, pe & dec, exp, inf & inst, ac, vo & inf, cut, ner, dig \\
\hline Asteraceae & $\begin{array}{l}\text { Crassocephalum montuosum (S. Moore) } \\
\text { Milne-Redh. }\end{array}$ & 4 & 5 & 1,2 & $\mathrm{f}, \mathrm{fl}$ & dec, exp & vo, bc, ac & ble, nut, gro, cult \\
\hline Asteraceae & Dichrocephala integrifolia Kuntze & 4 & 13 & 3,2 & pe, $f$ & dec, inf, exp & inst, ac, vo & $\begin{array}{l}\text { dig, imf, san, res, ner, } \\
\text { cult, gen }\end{array}$ \\
\hline Caryophyllaceae & Drymaria cordata Willd. ex Schult. & 4 & 8 & 2,0 & f, pe & dec, exp & vo, mas, ac & res, gen, mus, dig \\
\hline Malvaceae & Hibiscus noldeae Baker f. & 4 & 11 & 2,7 & f & exp, inf, dec & vo, scar, mas, ac & $\begin{array}{l}\text { cut, imf, ble, nut, gen, } \\
\text { gro, cult }\end{array}$ \\
\hline Simaroubaceae & Kirkia acuminata Oliv. & 4 & 6 & 1,5 & f, e & dec, exp & vo, ac & cut, san, gen, gro, dig \\
\hline Plantaginaceae & Plantago palmata Hook. f. & 4 & 12 & 2,9 & f, pe & dec, inf & vo & $\begin{array}{l}\text { inf, gen, cir, ner, poi, } \\
\text { mus, cult, dig }\end{array}$ \\
\hline Lamiaceae & Pycnostachys erici-rosenii R.E. Fr. & 4 & 9 & 2,2 & $\mathrm{f}, \mathrm{fl}, \mathrm{gr}, \mathrm{r}$ & $\begin{array}{l}\text { dec, exp, } \\
\text { cen, exp }\end{array}$ & vo, scar, inst, inh & sen, cir, ner, gro \\
\hline Polygonaceae & Rumex bequaertii De Wild. & 4 & 4 & 1,0 & $f, r$ & dec, sp, exp & vo, inst & inf, res, sen, ner \\
\hline Euphorbiaceae & Tragia Brevipes Pax & 4 & 9 & 2,2 & $f, r$ & dec, inf & vo, bs & nut, gen, gro \\
\hline
\end{tabular}

- Valeurs d'accessibilité : catégories 1, 2, 3 et 4 représentant les types d'habitats à accessibilité croissante (1 = accessibilité très faible, espèces de forêt primaire ; 2 = accessibilité faible, espèces de forêt secondaire ; 3 = accessibilité moyenne, espèces plantées ; 4 = accessibilité forte, espèces rudérales).

- Fréquence absolue d’usage : nombre de fois absolu où la plante a été mentionnée par les tradipraticiens, indépendamment de la catégorie d’usages. La valeur varie ici de 1 à 15.

- Fréquence relative d’usage : proportion de chaque plante mentionnée par les tradipraticiens, indépendamment de la catégorie d’usages.

- Parties utilisées : f : feuilles ; e : écorce ; fl : fleurs ; s : sève ; r : racines ; pe : plantes entières ; gr : graines ; t : tige ; no : noix.

- Modes de préparation : exp : expression ; dec : décoction ; pou : poudre ; inf : infusion ; cen : cendre ; mac : macération ; sp : sans préparation.

- Voies d’administration : vo : voie orale ; ac : application cutanée ; inst : instillations ; inh : inhalation ; bc : bain corporel ; scar : scarification ;

mas : massage ; asp : aspersion; bs : bain de siège ; bv : bain de vapeur ; bb : bain buccal.

- Catégorie de maladies : cf. tableau II. 
Tableau II.

Maladies appartenant à chaque catégorie, avec nombre de mentions ;

les sommes des nombres de mentions par catégorie sont indiquées entre parenthèses.

Catégories de maladies

Infections (Inf, 122)

Troubles de l'appareil circulatoire

(Cir, 17)

Troubles de l'appareil digestif

(Dig, 130)

\section{Troubles génito-urinaires(Gen, 89)}

Inflammations (Imf, 29)

Blessures et plaies (Ble, 19)

Troubles métaboliques (Met, 10)

Troubles musculaires (Mus, 42)

Troubles nerveux (Ner, 81)

Poison (Poi, 33)

Troubles de grossesse $(\mathrm{Gro}, 68)$

Troubles de l'appareil respiratoire

(Res, 29)

Troubles sensoriels (Sen, 14)

Troubles cutanés (Cut, 47)

Troubles du sang et tumeurs

(Sang, 29)

Troubles nutritionnels (Nut, 9)

Syndromes mal définis (Sym, 10)

Syndromes culturels (Cult, 60)
Pathologies

Amibiase (Nzokankulu*)

Candidose buccale (Chaminyagu*)

Choléra (Mukunguru*)

Immunodéficience (Muzirho*)

Tuberculose (Chigoholo ch'ikulu*)

Éléphantiasis (Birimbo*)

Amygdalite (Bilimi**)

Appendicite (Kidoletumbu**)

Douleur abdominale (Mukungulo*)

Constipation (Kurhanya*)

Diarrhée (Mushole*)

Salpingite (Mwanamimba**)

Gonorrhée (Chikagasi*)

Cystite (Buganga*)

Dysménorrhée Persis-tante

(Ndwala g'omuko*)

Brûlures (Muliro*)

Néphrite (Nfiko**)

Plaie (Chihulu*)

Diabète (Chisukari*)

Lombalgie (Omugongo*)

Maux de tête (Irhwe kuluma*)

Épilepsie (Lungungu*)

Empoisonnement (Oboge*)

Dystocie (Ukurhagwisa*)

IVG (Kukulaizimi*)

Angine (Bigoga*)

Asthme (Obuhema*)

Coryza (Kufuneka*)

Cataracte (Nshongo*)

Abcès (Muhama*)

Dermatose (Kuyaga*)

Furonculose (Mahurehure*)

Anémie (Kubulaomuko*)

Splénomégalie (Lusingu*)

Anorexie (Kurhahasha kulya*)

Douleur corps entier

(Mubirikuluma*)

Kivubo (Kivubo**)

Iseke (Iseke ${ }^{\star \star}$ )

Kunde (Kunde ${ }^{\star \star}$ )
M. Pathologies

41 Fièvre (Ihoma*)

Gastroentérite (Kadurha*)

3 Hépatite (Budiku*)

10 Helminthiase (Nzoka y’e nda*)

4

Tension artérielle

(Ndwala yo murhima*)

5 Diarrhée sanguinolente

(Kunya omuko*)

2 Hyper-gastralgie (Lurholero lukulu*) 18

4 Hypo-gastralgie (Lurholero lurho*)

2 Ulcère-gastralgie (Lurholerolwe chihulu*)

13

8 Frigidité (Kumasha*)

5 Disfonctionnement sexuel

(Kurhasha obuhya*)

6 Hydrocèle (Mishiha*)

2 Prostate (Kibofu**)

Nerf sciatique (Ihasha*)

Plaies ulcéreuses (Lukero*)

Fracture (Buvune*)

Folie (Isirhe*)

$\left.e^{\star}\right)$

.

Morsure de serpent (Kajokajoka*)

Hypogalactie (Nkumbwa*)

Menace d'avortement (Lumomyo*)

Toux (Chikoholo*)

Mal de poitrine (Kashiha*)

Conjonctivite (Ndwala ya masu*)

Kyste (Muziha*)

Mycose (Lubenja*)

6

8

Tumeur du sein

(Mokomoko*, Mpanga*)

11

3 Malnutrition (Obwaki*)

3 Vertige (Nzunguzungu*)

4 Malédiction (Mugereko*)

2 Mauvais esprit (Mudorho*)

2 Mpivu (Mpivu*)

13

9

M. Pathologies

2 Jaunisse (Ensiko*)

8 Lèpre (Bibenzi`)

16 Gingivite (Ndwala y’ekanwa*)

17 Téniasis $\left(\right.$ Tegu $^{\star \star}$ )

16 Hernie discale (Omugongo*)

16

8 Maux de dent (Ndwalay’aminu*) 4

4 Diarrhée avec douleur et sang 14

(Mukunguru*)

28 Hémorroïde (Kukunuka*) 4

2 Prolapsus utérin (Ibanzi*) 19

18 Infertilité masculine

(Kugumbalume*)

6 Infertilité féminine (Kugumbakazi*) 9

2 Prurit vaginal (Chilondatumbu*) 10

2 Rhumatisme (Kugogombaemisi*) 16

35 Foulure (Kuteguka*)

30 Migraine (Fumba*)

5

15 Fontanelle (Lukunga*)

23 Mort fœtale (Chibolwe*)

6 Pneumonie (Mwijimbwe*)

6 Sinusite (Muzerezi*)

M.

2 


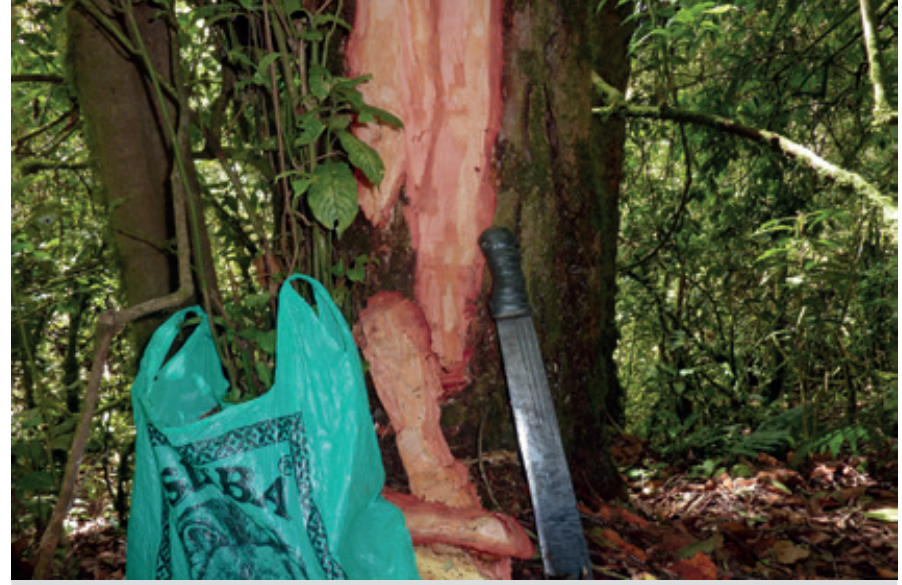

Photo 2a.

Écorçage de Carapa grandiflora Sprague (Meliaceae). Photo C. Shalukoma.

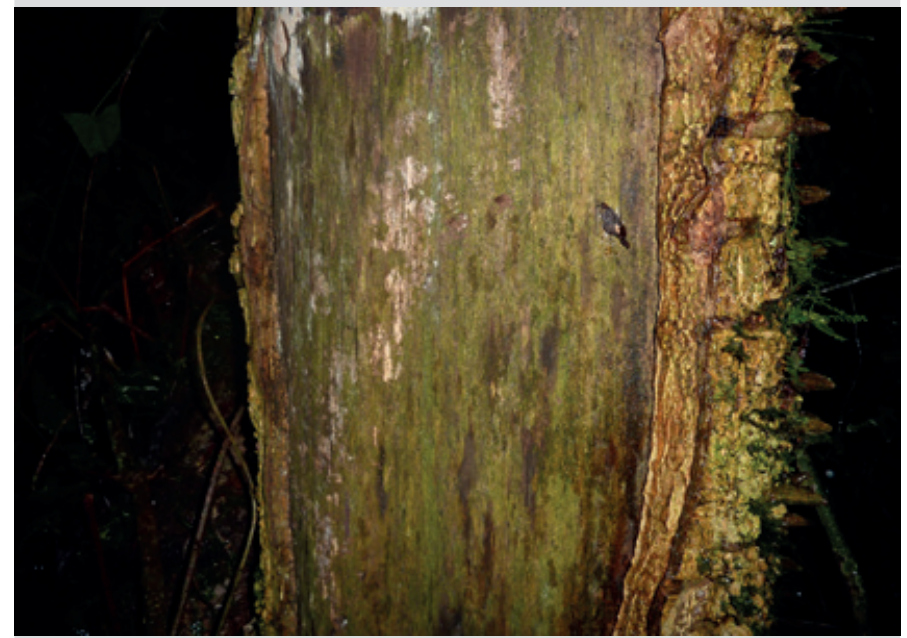

Photo 2 b.

Écorçage ancien de Zanthoxylum gilletii Oliv. (De Wild.) P. G. Waterman (Rutaceae).

Photo C. Shalukoma.

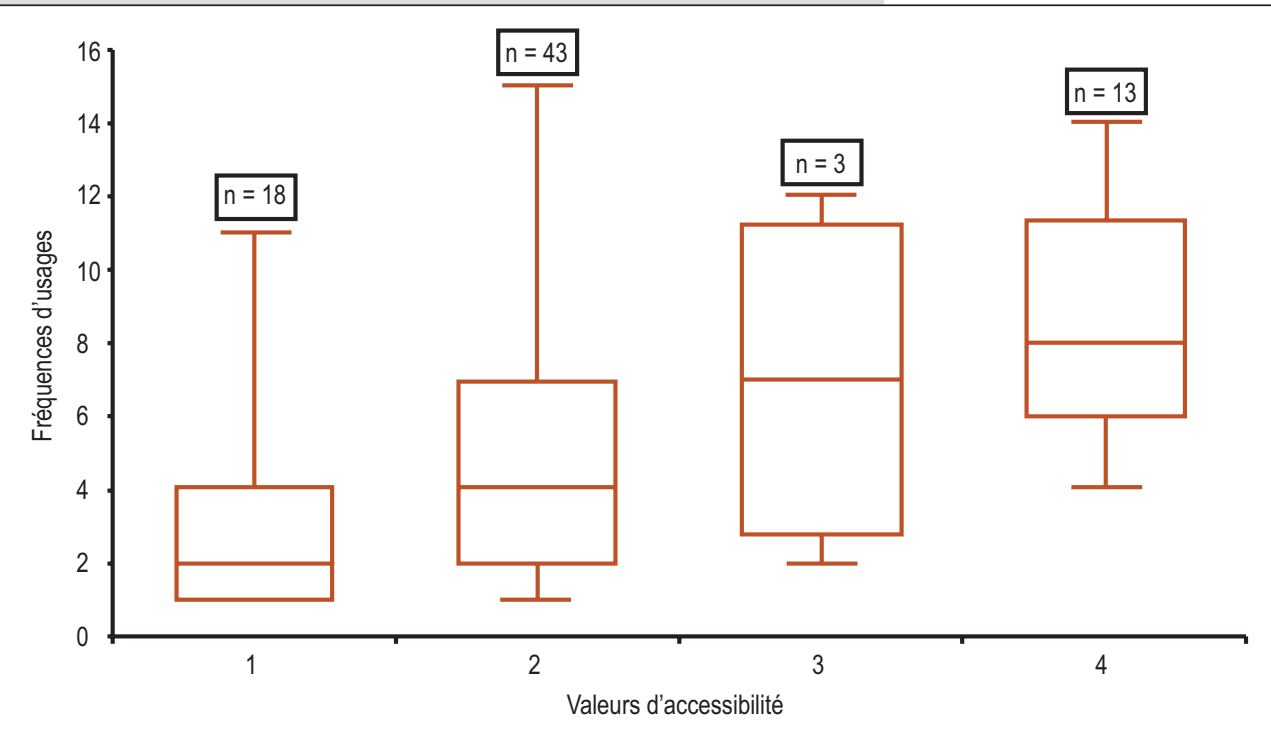

Figure 2.

Fréquences d'usage des espèces en fonction de leur accessibilité. La boîte à moustache représente l'intervalle dans lequel sont groupés $50 \%$ des fréquences des plantes.

La barre à l'intérieur est la fréquence médiane, la barre basse est la fréquence minimale et la barre haute est la fréquence maximale. Les valeurs de 1 à 4 représentent les échelles d'accessibilité ( 1 = accessibilité très faible, espèces de forêt primaire ; 2 = accessibilité faible, espèces de forêt secondaire ; 3 = accessibilité moyenne, espèces plantées ; 4 = accessibilité forte, espèces rudérales) ; $\mathrm{n}=$ nombre des plantes par accessibilité. la mort dans un délai très court.

Les espèces des jachères ou cultivées sont peu associées entre elles. Les espèces utilisées dans plus de dix recettes représentent $16 \%$ du total des espèces inventoriées. Une recette destinée à traiter le Mulonge, maladie de la catégorie des syndromes culturels, est composée de 14 plantes, toutes forestières. Cette maladie est décrite par les tradipraticiens comme un mauvais sort particulier faisant gonfler une partie du corps avec des douleurs atroces, provoquant

Pour 96 maladies identifiées, 18 catégories ont été établies avec un total de 838 mentions (tableau II). Les catégories de maladies avec plus de 80 mentions étaient les troubles digestifs (16\%), les maladies infectieuses (15\%), les troubles génito-urinaires (11\%) et les troubles nerveux (10\%).

\section{Accessibilité et fréquences d'usage des plantes médicinales}

Globalement, la fréquence d'usage des plantes rudérales et des jardins ainsi que leur accessibilité sont élevées par rapport aux plantes forestières (figure 2). Le test de Kruskal-Wallis montre une différence significative entre les plantes des quatre niveaux d'accessibilité $(H=17,64 ; p<0,001)$. Néanmoins, parmi les espèces forestières, Carapa grandiflora Sprague et Syzygium cordatum Hochst. ont présenté des fréquences d'usage très élevées pour les forêts primaires, tandis que Maesa lanceolata Forssk., Tabernaemontana johnstonii (Stapf) Pichon, Mikania cordata (Burm. f.) et Trema orientalis Blume ont des fréquences d'usage très élevées parmi les espèces de forêt secondaire. Les quantiles supérieurs très élevés sont expliqués par les fréquences de ces dernières espèces.

\section{Consensus d'informateurs et fidélité de plantes médicinales}

Un total de $50 \%$ des catégories de maladies ont donné lieu à un facteur de consensus d'informateurs élevé $\left(F_{c i}>0,70\right)$, particulièrement les troubles musculo-squelettiques $\left(F_{c i}=0,83\right)$ et les troubles infectieux $\left(F_{c i}=0,80\right)$. Les catégories de maladies induisant un $F_{c i}$ moyen compris entre 0,70 et 0,50 représentent $39 \%$, et $11 \%$ induisent un faible $F_{c i}<0,50$ (tableau III). Les catégories de maladies à $F_{c}$ élevé ont chacune une espèce à fidélité élevée tandis que celles à $F_{c i}$ moyen et faible ont entre 1 et 4 espèces fidèles.

À titre d'exemple, le taux de consensus de la catégorie d'usage musculo-squelettique a été calculé comme suit : 
$F_{c i}=n_{r}-n_{t} / n_{r}-1$

$F_{c i}=42-8 / 42-1$

$F_{c i}=0,83$

Avec $n_{r}=42$, le nombre des mentions des maladies de la catégorie musculo-squelettique ; $n_{t}=8$, le nombre total des mentions des plantes utilisées pour soigner les maladies identifiées dans la catégorie musculo-squelettique. La corrélation de Spearman a également montré que les taux de consensus ont tendance à être élevés quand les mentions sont élevées (figure 2). Cette corrélation positive ( $r s=0,50$; $p=0,03)$ suggère que plus une catégorie de maladies est traitée, plus les espèces indiquées sont utilisées.
Les taux de fidélité des plantes aux catégories de maladies se sont situés entre $2 \%$ et $41 \%$. Uniquement 15 espèces, représentant $19 \%$ des plantes inventoriées, ont une fidélité maximale pour toutes les catégories de maladies (tableau III). De ces espèces à fidélité maximale, 12 sont forestières. Un total de 10 espèces ont des indices de fidélité d'au moins $20 \%$ aux catégories de maladies, particulièrement Maesa lanceolata Forssk. (IF = 41 ; IF $=27$ aux troubles musculo-squelettiques et poisons) ; Gouania longispicata Engl. (IF = 40 aux troubles nutritionnels); Bidens pilosa $\mathrm{L}$. (IF $=40$ aux troubles sensoriels) et Hibiscus noldeae Baker f. (IF= 33 aux blessures).

\section{Tableau III.}

Facteur de consensus des tradipraticiens $\left(F_{c}\right)$ sur l'usage des plantes médicinales et les plantes ayant eu les taux de fidélité (\%) les plus élevés par catégorie de maladies.

\begin{tabular}{|c|c|c|c|c|c|c|c|c|c|c|c|c|c|c|c|c|c|c|}
\hline Catégorie de maladies & Mus & Nut & Sen & Ble & Poi & Imf & Res & Sym & Met & Cut & Inf & San & Cir & Gro & Dig & Ner & Cult & Gen \\
\hline $\begin{array}{l}\text { Nombre plantes } \\
\text { /catégorie }\end{array}$ & 8 & 4 & 4 & 6 & 10 & 13 & 8 & 5 & 10 & 14 & 25 & 14 & 11 & 22 & 33 & 23 & 24 & 28 \\
\hline $\begin{array}{l}\text { Nombre de mentions } \\
\text { /catégorie }\end{array}$ & 42 & 9 & 14 & 19 & 33 & 29 & 29 & 10 & 10 & 47 & 122 & 29 & 17 & 68 & 130 & 81 & 60 & 89 \\
\hline$F_{c} /$ catégorie & 0,83 & 0,63 & 0,77 & 0,72 & 0,72 & 0,57 & 0,75 & 0,56 & 0,33 & 0,72 & 0,80 & 0,54 & 0,38 & 0,69 & 0,75 & 0,73 & 0,61 & 0,69 \\
\hline Espèces & et le & fidé & is ma & males & par ca & gorie & $\begin{array}{l}\text { ice de } \\
\text { e mal }\end{array}$ & $\begin{array}{l}\text { idélité } \\
\text { dies }\end{array}$ & Catég & & & & & & & & & \\
\hline Maesa lanceolata & 41 & 0 & 0 & 0 & 27 & 0 & 0 & 0 & 0 & 0 & 0 & 0 & 0 & 0 & 0 & 2 & 4 & 0 \\
\hline Gouania longispicata & 0 & 40 & 0 & 0 & 0 & 0 & 0 & 0 & 14 & 0 & 0 & 0 & 0 & 6 & 0 & 0 & 0 & 4 \\
\hline Bidens pilosa & 0 & 0 & 40 & 0 & 7 & 7 & 0 & 20 & 0 & 0 & 0 & 7 & 0 & 6 & 3 & 5 & 4 & 0 \\
\hline Hibiscus noldae & 0 & 20 & 0 & 33 & 0 & 7 & 0 & 0 & 0 & 10 & 0 & 0 & 0 & 3 & 0 & 0 & 4 & 4 \\
\hline Basella alba & 0 & 0 & 0 & 0 & 0 & 20 & 0 & 0 & 0 & 0 & 3 & 0 & 0 & 3 & 0 & 0 & 0 & 0 \\
\hline Lobelia gibberoa & 0 & 0 & 0 & 0 & 0 & 0 & 20 & 0 & 0 & 0 & 0 & 0 & 0 & 0 & 0 & 0 & 0 & 0 \\
\hline Ageratum conyzoides & 0 & 0 & 0 & 0 & 0 & 7 & 0 & 20 & 0 & 0 & 0 & 0 & 0 & 6 & 2 & 0 & 4 & 0 \\
\hline Baissea multiflora & 0 & 0 & 0 & 11 & 0 & 7 & 10 & 20 & 0 & 0 & 3 & 0 & 0 & 0 & 0 & 0 & 0 & 4 \\
\hline Milicia excelsa & 0 & 0 & 0 & 0 & 0 & 7 & 0 & 20 & 0 & 0 & 0 & 0 & 0 & 0 & 0 & 0 & 0 & 0 \\
\hline Moringa oleifera & 0 & 0 & 0 & 0 & 0 & 0 & 0 & 0 & 14 & 0 & 0 & 0 & 9 & 0 & 0 & 0 & 0 & 0 \\
\hline Harungana montana & 0 & 0 & 0 & 0 & 0 & 0 & 0 & 0 & 14 & 5 & 3 & 13 & 0 & 0 & 5 & 0 & 0 & 2 \\
\hline $\begin{array}{l}\text { Entandrophragma } \\
\text { excelsum }\end{array}$ & 0 & 0 & 0 & 0 & 0 & 0 & 0 & 0 & 14 & 0 & 3 & 7 & 0 & 0 & 3 & 0 & 0 & 2 \\
\hline Parinari excelsa & 0 & 0 & 0 & 0 & 0 & 0 & 0 & 0 & 14 & 0 & 3 & 0 & 0 & 0 & 2 & 2 & 0 & 0 \\
\hline Ipomoea involucrata & 0 & 0 & 0 & 0 & 0 & 0 & 0 & 0 & 0 & 14 & 0 & 0 & 0 & 0 & 0 & 0 & 0 & 0 \\
\hline $\begin{array}{l}\text { Tabernaemontana } \\
\text { jonstonii }\end{array}$ & 0 & 0 & 0 & 0 & 0 & 0 & 0 & 0 & 0 & 5 & 14 & 0 & 0 & 6 & 6 & 0 & 4 & 2 \\
\hline
\end{tabular}

Catégorie de maladies (voir tableau II), nombre de mentions des maladies, nombre de mentions des plantes, indices de consensus des tradipraticiens. Les chiffres à l'intersection des plantes et des catégories de maladies sont les indices de fidélité par plante pour la catégorie. Les chiffres en rouge représentent les indices de fidélité les plus élevés des plantes par catégorie de maladies. 


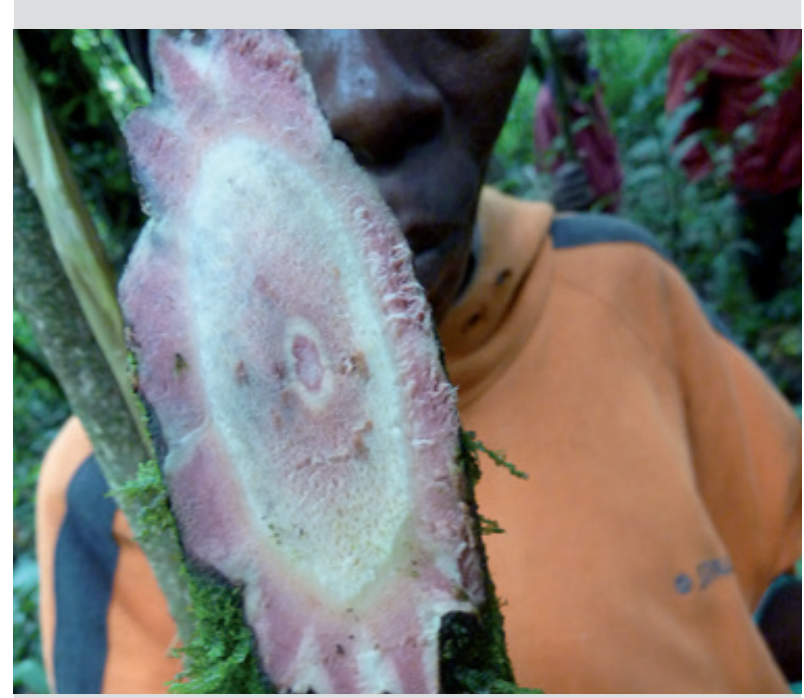

Photo 3.

Une femme Pygmée Twa, tradipraticienne de Kabare-

Miti avec un tronc de liane Gouania longispicata Engl.

(Rhamnaceae), pour les usages médicinaux.

Photo C. Shalukoma.

\section{Discussion}

\section{Plantes médicinales et pratiques médicales associées}

L'écorçage des plantes concerne les troncs d'arbres et parfois les racines. C'est une pratique qui interpelle car elle est particulièrement visible sur des espèces ligneuses spécifiques du PNKB. L'écorce de Prunus africana (Hook. f.) Kalkm. fait localement l'objet d'un commerce illicite. Selon Traoré et al. (2011), l'écorçage est souvent responsable des troubles physiologiques de l'arbre ; en outre, le prélèvement abusif des racines est fatal pour la plante. Betti (2001) et Nzuki et al. (2013) soulignent que les pratiques susmentionnées sont les plus courantes en Afrique, particulièrement en Afrique centrale. Au sein de la forêt du PNKB, le mode d'écorçage des arbres traduit que les écorces sont massivement prélevées. Ceci s'expliquerait entre autres par le fait qu'une fois séchées, elles se conservent plus longtemps. Les stocks constitués réduiraient les fréquences d'entrée des tradipraticiens dans cette aire protégée où ils s'exposent aux poursuites judiciaires. En revanche, en Éthiopie, les tradipraticiens préfèrent l'usage des plantes fraîches car celles-ci sont supposées avoir une grande efficacité thérapeutique (Lulekal et al., 2008). La préparation de solutions aqueuses ainsi que l'administration de drogues par voie orale sont les modes les plus utilisés chez les tradipraticiens autour du PNKB. Betti (2001) confirme qu'elles constituent les pratiques les plus répandues en Afrique. Selon Mussarat et al. (2014), les solutions aqueuses auraient prouvé leur efficacité. Mais elles connaissent des problèmes de conservation du fait que les tradipraticiens ne disposent pas de méthodes aseptiques pour les conserver (Betti, 2001).

Autour du PNKB, les recettes préparées à base d'une seule plante sont dominantes. Cette caractéristique des recettes a été également observée dans d'autres régions d'Afrique, notamment au Kenya (Kareru et al., 2007) et au Rwanda (Mukazayire et al., 2011 ; Kamagaju et al., 2013). Par contre, le principe de " médicaments complexes pour les maux complexes » guide souvent les pratiques traditionnelles à travers les médications multiplantes (Teklehaymanot et al., 2006). Cependant, certains auteurs ont lié la recette multiplantes au caractère secret de la médecine traditionnelle. Selon Schulz et al. (2001), pour une indication thérapeutique donnée, le tradipraticien peut citer plusieurs plantes pour dissimuler celle qui renferme le principe

actif. Pour Soengas (2010), le caractère secret de la médecine traditionnelle peut se justifier par la peur de la perte de pouvoir et l'intérêt économique. Dans le cas de cette étude où le consensus ethnobotanique est élevé, la caractéristique des recettes préparées à base d'une plante constituerait l'expression d'un savoir-faire des tradipraticiens, ce qui suppose un savoir traditionnel qui est maîtrisé et partagé au fil du temps.

\section{Accessibilité et fréquences d'usage des plantes médicinales}

Autour du PNKB, les espèces médicinales rudérales et ubiquistes sont les plus accessibles aux tradipraticiens. Elles sont généralement bien connues et l'accès est sans contraintes, contrairement aux espèces médicinales forestières qui, elles, semblent être moins populaires et moins accessibles. Pirker et al. (2012) ont démontré que l'accessibilité des plantes influence leurs usages au sein des populations et que les espèces médicinales ubiquistes semblent être continuellement utilisées du fait qu'elles restent accessibles.

Bien que les espèces forestières soient d'une faible accessibilité, certaines d'entre elles ont des fréquences d'usage très élevées. Nous citons Maesa lanceolata Forssk., Mikania cordata (Burm. f.) B. L. Rob et Trema orientalis Blume qui sont les espèces les plus prélevées en forêts secondaires pour le traitement des troubles musculo-squelettiques, tandis que Carapa grandiflora Sprague et Syzygium cordatum Hochst. ex C. Krauss sont des espèces des forêts primaires, prélevées pour traiter principalement les troubles des systèmes digestif et génito-urinaire. Au Kahuzi-Biega, les forêts secondarisées sont généralement localisées non loin des limites du Parc, des routes et des pistes pédestres le traversant. Vandebroek et al. (2004) avaient déjà démontré en Bolivie que les communautés vivant à proximité des forêts ont une large connaissance des espèces forestières. Ceci montre que les espèces forestières sont bien connues et accessibles par les tradipraticiens spécialisés.

\section{Consensus d'informateurs et fidélité des plantes médicinales}

Lorsqu'il est élevé, le facteur de consensus d'informateurs reflète une bonne connaissance des plantes médicinales, un savoir collectif de leurs usages, mais également un échange d'informations entre les tradipraticiens (Heinrich et al., 1998 ; Treyvaud-Amiguet et al., 2005 ; Gazzaneo et al., 2005). Certains auteurs ont qualifié de "secrète " la médecine traditionnelle des spécialistes (Baerts et Lehmann, 1989 ; Schulz et al., 2001). Ce caractère secret est indéniable. Il constitue un des facteurs d'érosion du savoir traditionnel (Collins et al., 2006 ; Soengas, 2010). Dans cette étude, lors des enquêtes de terrain, les tradipraticiens ont exigé des entretiens individuels et non en groupes. Lors de la récolte des herbiers en forêt, ils ont également demandé que les groupes soient constitués selon les affinités parentales ou les relations d'amitié. Ces deux faits laissent penser que la médecine traditionnelle dans notre zone d'étude revêt effectivement un caractère secret. Cependant, le facteur de consensus des tradipraticiens élevé pour plus de la moitié des catégories de maladies suggère que les échanges d'informations opèrent entre les tradipraticiens spécialistes autour du Parc. Les plantes les plus utilisées ne sont pas toutes efficaces. Certaines sont simplement connues et accessibles par beaucoup des tradipraticiens, telles Aloe barbadensis Mill. et Bidens pilosa L. qui semblent être des plantes multithérapeutiques, exotiques et de plus ubiquistes. 
Les troubles digestifs et les infections sont les catégories de maladies les plus mentionnées dans la zone étudiée. La qualité précaire de l'eau et la mauvaise hygiène dans l'utilisation des aliments expliqueraient en partie la prévalence de ces deux catégories de maladies et constituent des problèmes communs à beaucoup de pays en développement (Collins et al., 2006 ; Molares et Ladi, 2009). Les tradipraticiens ayant soigné les troubles nerveux ont souvent mentionné la folie comme une conséquence des conflits armés et de l'insécurité permanente dans la zone. Cette situation particulière a été soulignée par Goret (2013) qui a révélé qu'au centre psychiatrique Sosame de Bukavu $20 \%$ des cas de consultations annuelles relèvent d'un suivi médical pour cause de folie.

La fidélité des plantes aux catégories de maladies a été globalement moins élevée, inférieure à $50 \%$. Ceci s'expliquerait par le fait que les tradipraticiens autour du PNKB ont montré une tendance à utiliser du matériel végétal issu des forêts. Or, les plantes forestières sont moins accessibles dans la zone. Elles sont principalement prélevées au sein du PNKB, une forêt légalement protégée. Ces plantes forestières sont donc moins populaires bien qu'elles reflètent le matériel de la médecine traditionnelle spécialisée. Les plantes communes sont, quant à elles, accessibles et peuvent atteindre un taux de fidélité allant jusqu'à $100 \%$, comme l'ont montré des études menées dans d'autres parties du monde, tels le Sénégal (Gueye et al., 2012) et la Turquie (Ugulu, 2012). En revanche, la fidélité élevée de certaines espèces forestières s'expliquerait par leur abondance dans l'habitat, surtout celles qui se trouvent dans les forêts secondaires accessibles. C'est le cas de Maesa lanceolata Forssk., fidélisée dans les catégories des troubles musculaires et le traitement de poisons. C'est une espèce rencontrée dans presque tous les habitats secondarisés en altitude au Kahuzi-Biega. Plusieurs espèces à faible fréquence ( $15 \%$ ) ont été fidèles aux différentes catégories de maladies. Cela sous-entend qu'elles sont connues mais rares dans l'environnement immédiat des tradipraticiens, surtout qu'il n'existe pas de marchés de plantes médicinales dans la zone. Il y aurait donc risque de perte de connaissances pour ces espèces (Musa et al., 2011).

\section{Conclusion et perspectives}

Cette étude a mis en évidence les pratiques médicales, le niveau de consensus entre les tradipraticiens autour des espèces médicinales, ainsi que la fidélité des espèces aux diverses catégories de maladies dans la zone de montagne de Kahuzi-Biega. Le consensus élevé des tradipraticiens et la fidélité des espèces aux catégories de maladies suggèrent une tendance à l'homogénéité des connaissances. Cela traduit une tradition médicale bien définie, malgré un contexte socio-politique susceptible de favoriser l'érosion de la biodiversité et des connaissances locales. Les résultats obtenus constituent une base pour de futurs travaux de recherche liés à l'évaluation des usages locaux et des connaissances thérapeutiques à des fins pharmacologiques. En perspective, des études ethnobotaniques quantitatives seraient intéressantes à mener dans des zones où l'on peut trouver un nombre significatif de tradipraticiens spécialisés. Elles permettraient d'analyser le niveau de consensus à large échelle, mais aussi de dégager l'impact des tradipraticiens sur les plantes citées afin d'estimer le niveau d'ancrage de la tradition médicinale dans la région.

\section{Remerciements}

Nous remercions l'Institut congolais pour la conservation de la nature, ses partenaires ainsi que la Coopération technique belge pour avoir soutenu ce travail depuis plusieurs années. Merci également à la Fondation de Meurs-François pour son soutien financier, au Pr S. Pochet et au Dr L. Mubalama pour leurs contributions à ce travail, et enfin aux tradipraticiens qui ont offert leur coopération avec enthousiasme.

\section{Références bibliographiques}

Albuquerque U. P., 2009. Quantitative ethno-medicine or quantification in ethno-medicine? Journal of Ethnobotany Research and Applications, 7: 1-3.

Baerts M., Lehmann J., 1989. Guérisseurs et plantes médicinales de la région des crêtes Zaïre-Nil au Burundi. Tervuren, Belgique, Musée royal d'Afrique centrale (MRAC), Annales Sciences économiques, 18, $214 \mathrm{p}$.

Balagizi I., Ratti E., Kambale V., 2007. Les plantes médicinales du Bushi. Gênes, Italie, A. Insieme al Terzo Mondo, $313 p$

Betti L., 2001. Usages traditionnels et vulnérabilité des plantes médicinales dans la Réserve du Dja et dans les marchés de Yaoundé, Cameroun. Thèse de doctorat, Université libre de Bruxelles, Belgique, $228 \mathrm{p}$.

CNOM (Conseil national de l'Ordre des médecins en République démocratique du Congo), 2015. [En ligne] http:// www.cnom-rdc.org (consulté le 02/02/2015).

Collins S., Martins X., Mitchell A., Teshome A., Arnason J. T., 2006. Quantitative ethnobotany of two east Timorese cultures. Economic Botany, 60 (4): 347-361.

Cook F. E. M., 1995. Economic botany data collection standard. Kew, Royaume-Uni, Royal Botanic Gardens, 156 p.

Defour G., 1995. Éléments d'identification de 400 plantes médicinales et vétérinaires du Bushi. Bukavu, Zaïre, Bandari, $125 \mathrm{p}$.

De Wasseige C., Flynn J., Louppe D., Hiol Hiol F., Mayaux P. (éds), 2014. Les forêts du bassin du Congo - État des Forêts 2013. Neufchâteau, Belgique, Weyrich, 325 p.

Friedman J., Yaniv Z., Dafni A., Palewith D., 1986. A preliminary classification of the healing potential of medicinal plants, based on a rational analysis of an ethno-pharmacological field survey among Bedouins in the Negave desert, Israel. Journal of Ethnopharmacology, 16 (2-3): 275-287.

Gazzaneo L. R., Lucena R. F., Albuquerque U. P., 2005. Knowledge and use of medicinal plants by local specialists in a region of Atlantic Forest in the state of Pernambuco (Northeastern Brazil). Journal of Ethnobiology and Ethnomedicine, 1: 1-9.

Giday M., Asfaw Z., Woldu Z., Teklehaymanot T., 2009. Medicinal plant knowledge of the Bench ethnic group of Ethiopia: an ethnobotanical investigation. Journal of Ethnobiology and Ethnomedicine, 5: 34-40.

Goret C., 2013. Au Sud-Kivu, la santé mentale est un véritable problème. Magazine Metro, $n^{\circ}$ 2839. [En ligne] www. metrotime.be/fr/ (consulté le 24/09/2013).

Gruca M., Cámara-Leret R., Macía M. J., Balslev H., 2014. New categories for traditional medicine in the Economic Botany Data Collection Standard. Journal of Ethnopharmacology, 155 (2): 1388-1392. 
Gueye M., Cisse A., Diatta C. D., Dio S., Koma S., 2012. Étude ethnobotanique des plantes utilisées contre la constipation chez les Malinké de la communauté rurale de Tomboronkoto, Kédougou (Sénégal). The International Journal of Biological and Chemical Sciences, 6 (2) : 773-781.

Heinrich M., Ankli A., Frei B., Weimann C., Stichero, 1998. Medicinal plants in Mexico: healer's consensus and cultural importance. Social Science and Medicine, 47: 1859-1871.

Hoffman B., Gallaher G., 2007. Importance indices in ethnobotany. Ethnobotany Research and Applications, 5: 201-218.

ICCN, 2009. Plan général de gestion du Parc national de Kahuzi-Biega, 2009-2019. Kinshasa, République démocratique du Congo, ICCN, 129 p.

Kamagaju L., Bizuru E., Minani V., Morandini R., Stévigny C., Ghanem G., Duez P., 2013. An ethnobotanical survey of medicinal plants used in Rwanda for voluntary depigmentation. Journal of Ethnopharmacology, 150: 708-717.

Kareru P. G., Kenji G. M., Gachanja A. N., Keriko J. M., Mungai G., 2007. Traditional medicines among the Embu and Mbeere peoples of Kenya. African Journal of Traditional Complementary and Alternative Medicine, 4: 75-86.

Lulekal E., Kelbessa E., Bekele T., Yineger H., 2008. An ethnobotanical study of medicinal plants in Mana Angetu District, southeastern Ethiopia. Journal of Ethnobiology and Ethnomedicine, 4: 1-10.

Malan D. F., Danho F. R., Kouakou L. K., 2015. Medicinal plants and traditional healing practices in ehotile people, around the aby lagoon (eastern littoral of Côte d'Ivoire). Journal of Ethnobiology and Ethnomedicine, 11: 21.

Mangambu M. J., Diggelen R., Mwanga Mwanga J. C., Ntahobavuka H., Malaisse F., Robbrecht E., 2012. Étude ethnoptéridologique, évaluation des risques d'extinction et stratégies de conservation aux alentours du Parc National de Kahuzi Biega (RD Congo). Revue Internationale de Géologie, de Géographie et d'Écologie Tropicales, 36 : 137-158.

Mc Cune B., Grace J. B., 2002. Analysis of ecological communities. Gleneden Beach, OR, USA, MjM Software Design, $304 \mathrm{p}$.

Molares S., Ladi A., 2009. Ethnobotanical review of the Mapuche medicinal flora: Use patter on a regional scale. Journal of Ethnopharmacology, 34: 75-80.

Mukazayire M. J., Minani V., Ruffo C. K., Bizuru E., Stévigny C., Duez P., 2011. Traditional phytotherapy remedies used in Southern Rwanda for the treatment of liver diseases. Journal of Ethnopharmacology, 138: 415-431.

Musa S., Fathelrhman E., Elsheikh A., Lubna A., Abdel L. E., Yagi S., 2011. Ethnobotanical study of medicinal plants in the Blue Nile State, South-eastern Sudan. Journal of Medicinal Plants Research, 5 (17): 4287-4297.

Mussarat S., Amber R., Tariq A., Adnan M., Naser M., Abdelsalam N. M., Ullah R., Bibi R., 2014. Ethnopharmacological assessment of medicinal plants used against livestock infections by the people living around Indus River. BioMed Research International, volume 2014, 1-14.

Nzuki B., Termote C., Kibungu K., Van Damme P., 2013. Identification et importance locale des plantes médicinales utilisées dans la région de Mbanza-Ngungu, République démocratique du Congo. Bois et Forêts des Tropiques, 316 : 63-78. [En ligne] http://bft.cirad.fr/cd/BFT 316 63-77.pdf
Pirker H., Haselmair R., Kuhn E., Schunko C., Vogl C. R., 2012. Transformation of traditional knowledge of medicinal plants: the case of Tyroleans (Austria) who migrated to Australia, Brazil and Peru. Journal of Ethnobiology and Ethnomedicine, 8: 44.

Phillips O. L., 1996. Some quantitative methods for analyzing ethnobotanical knowledge. In: Alexiades M. N. (ed.). Selected Guidelines for Ethnobotanical Research. A Field Manual. New York, USA, New York Botanical Garden, Advances in Economic Botany 10.

Prota4U, Plant Resources of Tropical Africa, 2015. [En ligne] http://database.prota.org/, consulté le 16/01/2015.

Schneider E., 1996. Contribution à l'étude de l'ethnobotanique et de la médecine traditionnelle du Bushi (Kivu, Zaïre). Anthropos, $91:$ 53-74.

Schulz V., Hansel R., Tyler V. E., 2001. Medicinal Plants, Phytomedicines, and Phytotherapy. Rational Phytotherapy: A Physician's Guide to Herbal Medicine. Heidelberg, Berlin, Allemagne, Springer, $39 \mathrm{p}$.

Soengas B., 2010. La subsistance des Pygmées Bakoya à l'épreuve de l'agriculture : dynamique des savoirs ethnobotaniques et des pratiques (Département de la Zadié, Ogooué-Ivindo, Gabon). Thèse de doctorat, Muséum national d'histoire naturelle, Paris, France, 354 p.

Sokal R. R., Rohlf F. J., 2012. Biometry: the principles and practice of statistics in biological research. 4th edition. New York, USA, W. H. Freeman and Co., 937 p.

Teklehaymanot T., Giday M., Medhin G., Mekonnen Y., 2006. Knowledge and use of medicinal plants by people around Debre Libanos Monastery in Ethiopia. Journal of Ethnopharmacology, 111: 271-283.

Traoré L., Ouédraogo I., Ouédraogo A., Thiombiano A., 2011. Perceptions, usages et vulnérabilité des ressources végétales ligneuses dans le Sud-Ouest du Burkina Faso. International Journal of Biological and Chemical Sciences, 5 (1) : 258-278.

Treyvaud-Amiguet V., Arnason J., Maquin P., Cal V., Sanchez-Vindaz P., Poveda L., 2005. A consensus ethnobotany of the Q'eqchi' Maya of southern Belize. Economic Botany, 59: 29-42.

Trotter R. T., Logan M. H., 1986. Informant consensus: A new approach for identifying potentially effective medicinal plants. In: Etkin N. L. (ed.). Plants in indigenous medicine and diet: biobehavioral approaches. Bedford Hills, NY, USA, Redgrave Publishing Company, 91-112.

Turner N. J, 1988. Ethnobotany of coniferous trees in Thompsons and Lillooet interior salish of British Colombia. Economic Botany, 42 (2): 177-194.

Ugulu I., 2012. Fidelity level and knowledge of medicinal plants used to make therapeutic turkish baths. Ethno Medicine Journal, 6 (1): 1-9.

Vandebroek I., Van Damme P., Van Puyvelde L., Arrazola S., De Kimpe N., 2004. A comparison of traditional healers' medicinal plant knowledge in the Bolivian Andes and Amazon. Social Science and Medicine, 59: 837-849.

Vandebroek I., Balick M. J., 2012. Globalization and Loss of Plant Knowledge: Challenging the Paradigm. PLoS One, 7 (5): e37643.

Wong J. L., Thornber K., Baker N., 2001. Évaluation des ressources en produits forestiers non ligneux. Expériences et principes de biométrie. Rome, Italie, FAO, Produits forestiers non ligneux, 13. 